\title{
Egalitarian-Equivalence and the Pareto Principle for Social Preferences*
}

\author{
Koichi Tadenuma ${ }^{\dagger}$ \\ This version: September 2002 \\ First version: July 2002
}

\begin{abstract}
When we construct social preferences, the Pareto principle is often in conflict with the equity criteria: there exist two allocations $x$ and $y$ such that $x$ Pareto dominates $y$, but $y$ is an equitable allocation whereas $x$ is not. The efficiency-first principle requires to rank an allocation $x$ higher than $y$ if (i) $x$ Pareto dominates $y$ or (ii) $x$ and $y$ are Pareto-noncomparable and $x$ is equitable whereas $y$ is not. The equity-first principle reverses the order of application of the two criteria. Adopting egalitarian-equivalence as the notion of equity, we examine rationality of the social preference functions based on the efficiency-first or the equity-first principle. The degrees of rationality vary widely depending on which principle is adopted, and depending on the range of egalitarian-reference bundles. We show several impossibility and possibility results as well as a characterization of the social preference function introduced by Pazner and Schmeidler (1978). We also identify the sets of maximal allocations of the social preference relations in an Edgeworth box. The results are contrasted with those in the case where no-envy is the notion of equity.

Joural of Economic Literature Classification Numbers: D71, D63, D61.
\end{abstract}

*This paper is a much extended version of a part of Tadenuma (1998). The author is grateful to Marc Fleurbaey, Serge-Chiristophe Kolm, Kotaro Suzumura, and William Thomson for helpful comments on the earlier version of the paper.

${ }^{\dagger}$ Faculty of Economics, Hitotsubashi University, Kunitachi, Tokyo 186-8601, Japan. Fax:(81)-42-580-8748. E-Mail: tadenuma@econ.hit-u.ac.jp 


\section{Introduction}

As is often criticized, the Pareto principle is silent about distributional equity. Several important notions of equitable allocations that depend only on ordinal individual preferences have been proposed and studied extensively in the theory of fair allocation. Among others, the concepts of no-envy and egalitarian-equivalence are central. ${ }^{1}$ In the literature of fair allocation, authors usually focus only on the "optimal" allocations in a feasible set. However, several notable attempts have been made to incorporate the ideas of distributional equity into construction of social orderings of all allocations, following the tradition of Arrovian social choice theory (Arrow, 1951). Such contributions include Feldman and Kirman (1974), Pazner and Schmeidler (1978), Gärdenfors (1978), Suzumura (1981a,b, 1996), Fleurbaey and Maniquet (2001), Fleurbaey (2001) and Tadenuma (2002).

It is important to construct a reasonable social ordering of all allocations, not just identifying "optimal" allocations. Indeed, it is quite rare in the real world that we could choose an "optimal" allocation among all feasible allocations, but rather, we are often faced with choice between several "nonoptimal" allocations.

A most natural way to incorporate the idea of equitable allocations would be to rank an allocation $x$ higher than an allocation $y$ if $x$ is equitable but $y$ is not. However, even this weakest requirement has to conflict with the Pareto principle: there exist two allocations $x$ and $y$ such that $x$ Pareto dominates $y$, but $x$ is not equitable while $y$ is. The fundamental conflict between efficiency and equity was first observed by Kolm (1971) in the case of no-envy as the concept of equity.

Faced with the conflict between efficiency and no-envy, Tadenuma (1998, 2002) proposed two contrasting principles to construct social preference relations: The efficiency-first principle requires to rank an allocation $x$ higher than $y$ if (i) $x$ Pareto dominates $y$ or (ii) $x$ and $y$ are Pareto-noncomparable and $x$ is envy-free whereas $y$ is not. The equity-first principle reverses the order of application of the two criteria.

In this paper, we focus on another important concept of equity, egalitarian-equivalence: an allocation $x$ is egalitarian-equivalent if there exists a consumption bundle $a_{0}$ such that every agent is indifferent between

\footnotetext{
${ }^{1}$ An envy-free allocation was introduced by Kolm (1971) and Foley (1967), and an egalitarian-equivalent allocation was proposed by Pazner and Schmeidler (1978).
} 
his consumption bundle at $x$ and the bundle $a_{0}$. The bundle $a_{0}$ is called an egalitarian-reference bundle. Just like Pareto efficiency and no-envy, this concept of equity depends only on ordinal preferences of individuals. We study rules to construct social preferences over all allocations, or simply social preference functions, based on the Pareto criterion and the equity criterion as egalitarian-equivalence.

First we observe a conflict between the two criteria: there exist allocations $x$ and $y$ such that $x$ Pareto dominates $y$ whereas $y$ is egalitarian-equivalent but $x$ is not. The two criteria are completely opposed to each other on which allocation is socially more desirable. Hence, in order to rank allocations, we need to give priority to one criterion over the other.

We examine rationality (or choice-consistency) properties of social preference functions based on either the efficiency-first principle or the equity-first principle. It is shown that there exists no social preference function satisfying the efficiency-first principle and acyclicity together if no restriction is imposed on the range of egalitarian-reference bundles. However, if we fix a ray from the origin, and take only egalitarian-reference bundles on the ray, then there exist social preference functions satisfying the efficiency-first principle and transitivity. An example is the social preference function proposed by Pazner-Schmeidler (1978), which has been characterized nicely by Fleurbaey (2001). Based on the efficiency-first principle, we present another characterization of the Pazer-Schmeidler social preference function. Turning to the equity-first principle, there exist social preference functions satisfying the equity-first principle and transitivity. The result holds whether the range of reference bundles is restricted or not. However, no social preference function satisfies these conditions and continuity together.

We also identify the maximal allocations of the social preference relations in an Edgeworth box. In contrast with the case where no-envy is the concept of equity, the set of maximal allocations of the coarsest efficiency-first relation always coincides with that of the coarsest equity-first relation, and the maximal set of any social preference relation based either of the two principles is a subset of this set.

The rest of the paper is organized as follows. The next section introduces notation and the basic concepts. In section 3, we formulate axioms for social preference functions, including ones that reflect the efficiency-first or the equity-first principle. Section 4 examines rationality (choice-consistency) of social preference functions. Maximal allocations in an Edgeworth are investigated in section 5. Section 6 contains concluding remarks. 


\section{Pareto and Egalitarian-Equivalence}

There are $n$ agents and $m$ infinitely divisible goods, where $n$ and $m$ are some finite numbers. Let $N=\{1, \cdots, n\}$ be the set of agents. Denoting by $\mathbb{R}$ the set of real numbers, the consumption set of each agent is $\mathbb{R}_{+}^{m}$. Let $\mathcal{R}$ be the class of preference relations on $\mathbb{R}_{+}^{m}$ that are reflexive, transitive, complete, continuous, and strictly monotonic. Each agent $i \in N$ is endowed with a preference relation $R_{i}$ in $\mathcal{R}$. The strict preference relation and the indifference relation associated with $R_{i}$ are denoted by $P_{i}$ and $I_{i}$, respectively. A list of preference relations, $\left(R_{i}\right)_{i \in N} \in \mathcal{R}^{n}$, is called a preference profile, and denoted by $R_{N}$.

An allocation is a vector $x=\left(x_{1}, \cdots, x_{n}\right) \in \mathbb{R}_{+}^{m n}$ where each $x_{i}=$ $\left(x_{i 1}, \cdots, x_{i m}\right) \in \mathbb{R}_{+}^{m}$ is a consumption bundle of agent $i \in N$. The set of all allocations is denoted by $X{ }^{2}$

Let $R_{N} \in \mathcal{R}^{n}$ be given. An allocation $x \in X$ Pareto dominates an allocation $y \in X$ if $x_{i} R_{i} y_{i}$ for all $i \in N$ and $x_{i} P_{i} y_{i}$ for some $i \in N$. If $x$ Pareto dominates $y$, we write $x \succ_{P} y$. An allocation $x$ strongly Pareto dominates an allocation $y$ if $x_{i} P_{i} y_{i}$ for all $i \in N$. If $x$ strongly Pareto dominates $y$, we write $x \succ_{S P} y$. Given a set $Z \subset X$, an allocation $x \in Z$ is Pareto efficient in $Z$ if there is no $y \in Z$ with $y \succ_{P} x$. Let $P(Z)$ be the set of Pareto efficient allocaitons in $Z$. An allocation $x \in X$ is egalitarianequivalent if there is a consumption bundle $a_{0} \in \mathbb{R}_{+}^{m}$ such that for all $i \in N$, $x_{i} I_{i} a_{0}$. Then, the bundle $a_{0}$ is called an egalitarian-reference bundle for $x$. For each $Z \subseteq X$, let $E(Z)$ denote the set of egalitarian-equivalent allocations in $Z$. We simply write $E(X)=E$.

A particular subset of egalitarian-equivalent allocations can be defined by restricting the range of reference bundles. A natural restriction may be to take only reference bundles that are proportional to some basic vector. Formally, given $\bar{r} \in \mathbb{R}_{++}^{m}$, an allocation $x \in X$ is said to be egalitarianequivalent for a fixed reference ray with $\bar{r}$ or simply $\bar{r}$-egalitarian-equivalent if there is a real number $\lambda \in \mathbb{R}_{+}$such that for all $i \in N, x_{i} I_{i} \lambda \bar{r}$. For each $Z \subseteq X$, let $E_{\bar{r}}(Z)$ be the set of $\bar{r}$-egalitarian-equivalent allocations. Let $E_{\bar{r}}(X)=E_{\bar{r}}$.

For all $x, y \in \mathbb{R}_{+}^{m n}$, if $x \in E$ and $y \notin E$, then we write $x \succ_{E} y$. Similarly, if $x \in E_{\bar{r}}$ and $y \notin E_{\bar{r}}$, then we write $x \succ_{E_{\bar{r}}} y$.

\footnotetext{
${ }^{2}$ We assume that $X=\mathbb{R}_{+}^{m n}$. However, we might impose a resource constraint and let $X$ be the set of all feasible allocations: given some fixed $\Omega \in \mathbb{R}_{+}^{m}, X=\left\{x \in \mathbb{R}_{+}^{m n} \mid \sum_{i \in N} x_{i} \leq\right.$ $\Omega\}$. All the results in this paper hold with this definition of $X$.
} 
Let $\mathcal{Q}$ be the set of reflexive binary relations on $X$. A social preference function is a mapping $f: \mathcal{R}^{n} \rightarrow \mathcal{Q}$. For each $R_{N} \in \mathcal{R}^{n}, \succeq=f\left(R_{N}\right)$ is called the social preference relation at $R_{N}$. In the following, we denote by $\succeq$ the social preference relation $f\left(R_{N}\right)$ at a given $R_{N} \in \mathcal{R}^{n}$, and by $\succ$ its asymmetric part. Given $\succeq$ and $Z \subset X$, an allocation $x \in Z$ is a maximal element in $Z$ for $\succeq$ if there is no $y \in Z$ such that $y \succ x$. Let $M(Z, \succeq)$ be the set of maximal elements in $Z$ for $\succeq$.

A social preference relation $\succeq^{\prime} \in \mathcal{Q}$ is said to be a refinement of $\succeq \in \mathcal{Q}$ if $\succ \subset \succ^{\prime}$ and $\succ \neq \succ^{\prime}$. If this is the case, we also say that $\succeq$ is coaser than $\succeq^{\prime}$. A social preference function $f$ is a refinement of a social preference function $g$ if for all $R_{N} \in \mathcal{R}^{n}, f\left(R_{N}\right)$ is a refinement of $g\left(R_{N}\right)$.

\section{Axioms for Social Preference Functions}

We formulate properties, or axioms for social preference functions, and examine the existence of a function satisfying the axioms.

The first two axioms have been playing a central role in welfare economics and social choice theory.

Pareto: For all $R_{N} \in \mathcal{R}^{n}$ and all $x, y \in X$, if $x \succ_{P} y$, then $x \succ y$.

Weak Pareto: For all $R_{N} \in \mathcal{R}^{n}$ and all $x, y \in X$, if $x \succ_{S P} y$, then $x \succ y$.

The next two axioms mean that if $x$ is equitable in the sense of egalitarianequivalence (or $\bar{r}$-egalitarian-equivalence) but $y$ is not, then $x$ should be regarded as socially more desirable than $y$.

Egalitarian-Equivalence: For all $R_{N} \in \mathcal{R}^{n}$ and all $x, y \in X$, if $x \succ_{E} y$, then $x \succ y$.

$\overline{\boldsymbol{r}}$-Egalitarian-Equivalence: There exists $\bar{r} \in \mathbb{R}_{+}^{m}$ such that for all $R_{N} \in$ $\mathcal{R}^{n}$ and all $x, y \in X$, if $x \succ_{E_{\bar{r}}} y$, then $x \succ y$.

There is no logical relation between Egalitarian-Equivalence and $\bar{r}$ Egalitarian-Equivalence. To check this, consider the social preference function $f$ define as follows: for all $R_{N} \in \mathcal{R}^{n}$, and all $x, y \in X, x \succeq y$ if and only if $x \in E$ or $y \notin E$. Given $\bar{r} \in \mathbb{R}_{+}^{m}$, define another social preference function $f^{\prime}$ as follows: for all $R_{N} \in \mathcal{R}^{n}$, and all $x, y \in X, x \succeq^{\prime} y$ if and 
only if $x \in E_{\bar{r}}$ or $y \notin E_{\bar{r}}$. Clearly, $f$ satisfies Egalitarian-Equivalence, and $f^{\prime}$ satisfies $\bar{r}$-Egalitarian-Equivalence. Choose $R_{N}, R_{N}^{\prime} \in \mathcal{R}^{n}$ such that there exist $x, y, z \in X$ with $x \in E_{\bar{r}}, y \in E$ but $y \notin E_{\bar{r}}$, and $z \notin E$. Then, although $x \succ_{E_{\bar{r}}} y, x \succ y$ does not hold. Hence, $f$ does not satisfy $\bar{r}$-EgalitarianEquivalence. On the other hand, $y \succ^{\prime} z$ does not hold despite $y \succ_{E} z$. Thus, $f^{\prime}$ does not satisfy Egalitarian-Equivalence.

As we will see later, the two axioms, Pareto and Egalitarian-Equivalence (or Pareto and $\bar{r}$-Egalitarian-Equivalence), conflict with each other. Hence, we propose to apply one criterion only conditionally on the other. First, we apply the equity criterion only when the Pareto criterion does not make strict orders.

P-Conditional Egalitarian-Equivalence: For all $R_{N} \in \mathcal{R}^{n}$ and all $x, y \in$ $X$, if $x \nsucc_{P} y, y \nsucc_{P} x$, and $x \succ_{E} y$, then $x \succ y$.

P-Conditional $\overline{\boldsymbol{r}}$-Egalitarian-Equivalence: For all $R_{N} \in \mathcal{R}^{n}$ and all $x, y \in X$, if $x \nsucc_{P} y, y \nsucc_{P} x$, and $x \succ_{E_{\bar{r}}} y$, then $x \succ y$.

The above two axioms may be strengthened by replacing $\left[x \nsucc_{P} y, y \nsucc_{P}\right.$ $x]$ with $\left[x \nsucc_{S P} y, y \nsucc_{S P} x\right]$ in the hypotheses. With this strengthening, however, the axioms become too strong to be compatible with Pareto. Hence, we only consider the above versions.

Symmetrically, we may apply the Pareto criterion only if the equity criterion does not make strict rankings.

E-Conditional Pareto: For all $R_{N} \in \mathcal{R}^{n}$ and all $x, y \in X$, if $x \nsucc_{E} y$, $y \nsucc_{E} x$, and $x \succ_{P} y$, then $x \succ y$.

$\mathbf{E}_{\overline{\boldsymbol{r}}}$-Conditional Pareto: For all $R_{N} \in \mathcal{R}^{n}$ and all $x, y \in X$, if $x \nsucc_{E_{\bar{r}}} y$, $y \nsucc_{E_{\bar{r}}} x$, and $x \succ_{P} y$, then $x \succ y$.

Another important requirement for social preference functions is rationality or choice-consistency in the sense of transitivity, quasi-transitivity, or acyclicity of the social preference relations generated by the functions.

Transitivity: For all $R_{N} \in \mathcal{R}^{n}$ and all $x, y, z \in X$, if $x \succeq y$ and $y \succeq z$, then $x \succeq z$.

Quasi-Transitivity: For all $R_{N} \in \mathcal{R}^{n}$ and all $x, y, z \in X$, if $x \succ y$ and $y \succ z$, then $x \succ z$. 
Acyclicity: For all $R_{N} \in \mathcal{R}^{n}$, all integer $k$ with $k \geq 3$, and all $x^{1}, \ldots, x^{k} \in$ $X$, if $x^{\ell} \succ x^{\ell+1}$ for all $\ell \in\{1, \ldots, k-1\}$, then $x^{k} \nsucc x^{1}$.

It is also desirable to have complete and continuous social preference relations.

Completeness: For all $R_{N} \in \mathcal{R}^{n}$ and all $x, y \in X, x \succeq y$ or $y \succeq x$.

Continuity: For all $R_{N} \in \mathcal{R}^{n}$ and all $x \in X$, both $\{y \in X \mid y \succeq x\}$ and $\{y \in X \mid x \succeq y\}$ are closed in $X$.

\section{Rationality of Social Preference Functions}

Our first result is incompatibility of the Pareto principle with EgalitarianEquivalence (or $\bar{r}$-Egalitarian-Equivalence).

Proposition 1 There exists no social preference function satisfying Weak Pareto and Egalitarian-Equivalence. There exists no social preference function satisfying Weak Pareto and $\bar{r}$-Egalitarian-Equivalence.

Proof. For simplicity, consider an economy with two agents $N=\{1,2\}$ and two goods 1,2. Let $f$ be a social preference function. Assume that $R_{1}=R_{2}$ and the preference relation is represented by the following utility function:

$$
\begin{aligned}
& u_{i}\left(x_{i 1}, x_{i 2}\right)=x_{i 1}+3 x_{i 2} \text { if } x_{i 2} \leq x_{i 1} \\
& u_{i}\left(x_{i 1}, x_{i 2}\right)=3 x_{i 1}+x_{i 2} \text { if } x_{i 2} \geq x_{i 1}
\end{aligned}
$$

Define $x=\left(x_{1}, x_{2}\right)=((8,2),(2,8)), y=\left(y_{1}, y_{2}\right)=((6,6),(4,4))$ and $\bar{r}=$ $(1,1)$. Then, the allocation $y$ strongly Pareto dominates the allocation $x$. Hence, if the social preference function $f$ satisfies Weak Pareto, then we must have $y \succ x$.

On the other hand, since $u_{1}\left(x_{1}\right)=u_{1}\left(\frac{7}{2} \bar{r}\right)$ and $u_{2}\left(x_{2}\right)=u_{2}\left(\frac{7}{2} \bar{r}\right)$, the allocation $x$ is $\bar{r}$-egalitarian-equivalent with $\frac{7}{2} \bar{r}$ being the reference bundle. However, since $R_{1}=R_{2}$ and $y_{1} \gg y_{2}$, there cannot exist $a_{0} \in X$ such that $y_{1} \quad I_{1} a_{0}$ and $y_{2} I_{2} a_{0}$. Hence, $y$ is not egalitarian-equivalent. Thus, if $f$ satisfies Egalitarian-Equivalence or $\bar{r}$-Egalitarian-Equivalence, then $x \succ y$ must hold. But we cannot have both $y \succ x$ and $x \succ y$.

From the above result, we have to rely on the conditional version of either the Pareto principle or the equity criterion. Next we examine rationality 
properties of social preference functions satisfying

(1) Pareto and P-Conditional $(\bar{r}$-)Egalitarian-Equivalence, or

(2) $\left(\bar{r}_{-}\right)$Egalitarian-Equivalence and $\left(\bar{r}_{-}\right)$E-Conditional Pareto.

If we accept Pareto as the first principle and Egalitarian-Equivalence as the second without imposing restrictions on the range of reference bundles, then even the minimal requirement of rationality cannot be met.

Proposition 2 There exists no social preference function satisfying Weak Pareto, P-Conditional Egalitarian-Equivalence, and Acyclicity. ${ }^{3}$

Proof. (See Figures 1 and 2.) Consider an economy with two agents $N=\{1,2\}$ and two goods 1,2 . Let $f$ be a social preference function satisfying Weak Pareto and P-Conditional Egalitarian-Equivalence. We show that there exists $R_{N} \in \mathcal{R}^{n}$ such that $\succeq=f\left(R_{N}\right)$ has a cycle.

Assume that $R_{1}$ is represented by the following piecewise linear utility function:

$$
\begin{aligned}
& u_{1}\left(x_{11}, x_{12}\right)=x_{11}+13 x_{12} \text { if } x_{12} \leq x_{11} \\
& u_{1}\left(x_{11}, x_{12}\right)=13 x_{11}+x_{12} \text { if } x_{12} \geq x_{11}
\end{aligned}
$$

and $R_{2}$ is represented by the following piecewise linear utility function:

$$
\begin{aligned}
u_{2}\left(x_{21}, x_{22}\right)= & x_{21}+13 x_{22} \\
& \quad \text { if } x_{22} \leq x_{21} \\
u_{2}\left(x_{21}, x_{22}\right)= & 7\left(x_{21}+x_{22}\right) \\
& \quad \text { if } x_{22} \geq x_{21} \text { and } x_{22} \leq 20 \\
u_{2}\left(x_{21}, x_{22}\right)= & \frac{7}{13}\left(13 x_{21}+x_{22}+240\right) \\
& \quad \text { if } x_{22} \geq 20 \text { and } x_{22} \leq-13 x_{21}+280 \\
u_{2}\left(x_{21}, x_{22}\right)= & 13 x_{21}+x_{22} \\
& \quad \text { if } x_{22} \geq x_{21} \text { and } x_{22} \geq-13 x_{21}+280
\end{aligned}
$$

Define $x, y, z, w \in X$ by $x=((19,9),(9,19)), y=((13,10),(15,18))$, $z=((24,10),(4,18))$ and $w=((17,15),(11,13)) .{ }^{4}$ Then, observe the following facts:

\footnotetext{
${ }^{3}$ This result was first reported in Tadenuma (1998).

${ }^{4}$ Notice that the total amounts of resources at each of the four allocations are the same. Hence, the result holds true even if we consider only feasible allocations with no free disposal under some resource constraint.
} 
$\operatorname{good} 2$

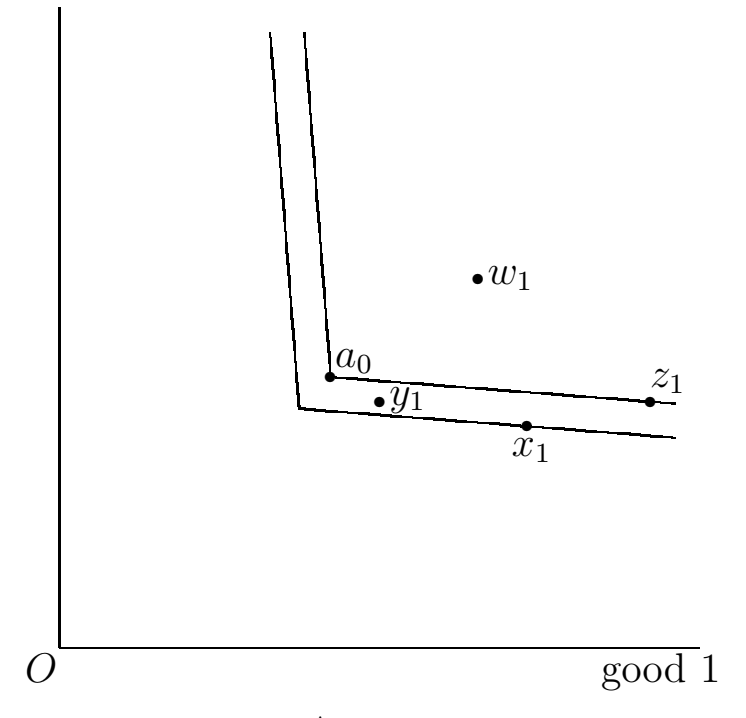

Agent 1 good 2

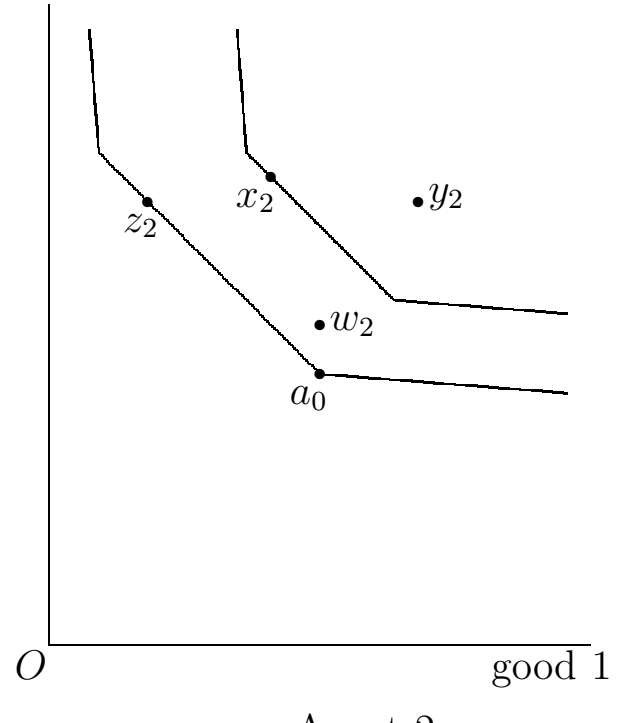

Agent 2

Figure 1: Preferences of Agent 1 and Agent 2

(1) The allocation $y$ strongly Pareto dominates the allocation $x$.

(2) Also, $w$ strongly Pareto dominates $z$.

(3) However, $y$ and $z$ are Pareto noncomparable.

(4) Similarly, $x$ and $w$ are Pareto noncomparable.

(5) On the other hand, the allocation $x$ is egalitarian-equivalent with the reference bundle $(9,19)$ since $u_{1}\left(x_{1}\right)=u_{1}(9,19)$ and $u_{2}\left(x_{2}\right)=u_{2}(9,19)$.

(6) Similarly, $z$ is egalitarian equivalent with the reference bundle $a_{0}=$ $(11,11)$.

(7) However, $y$ is not egalitarian-equivalent. This can be checked by noting that:

(i) $y_{1} I_{1} t$ if and only if $\left[t_{2}=-13 t_{1}+143\right.$ and $\left.0 \leq t_{1} \leq \frac{143}{14}\right]$ or $\left[t_{2}=-\frac{1}{13} t_{1}+11\right.$ and $\left.t_{1} \geq \frac{143}{14}\right]$, and

(ii) $y_{2} I_{2} t$ if and only if $\left[t_{2}=-13 t_{1}+189\right.$ and $\left.0 \leq t_{1} \leq 13\right]$ or $\left[t_{2}=-t_{1}+33\right.$ and $\left.13 \leq t_{1} \leq \frac{33}{2}\right]$ or $\left[t_{2}=-\frac{1}{13} t_{1}+\frac{231}{13}\right.$ and $\left.t_{1} \geq \frac{33}{2}\right]$.

Hence, there exists no $t \in \mathbb{R}_{+}^{2}$ such that $y_{1} I_{1} t$ and $y_{2} I_{2} t$. (Figure 2)

(8) Similarly, it can be shown that $w$ is not egalitarian-equivalent.

Since $f$ satisfies Weak Pareto, it follows from (1) that $y \succ x$. Because $f$ satisfies P-Conditional Egalitarian-Equivalence, by (3), (6) and (7), we must 


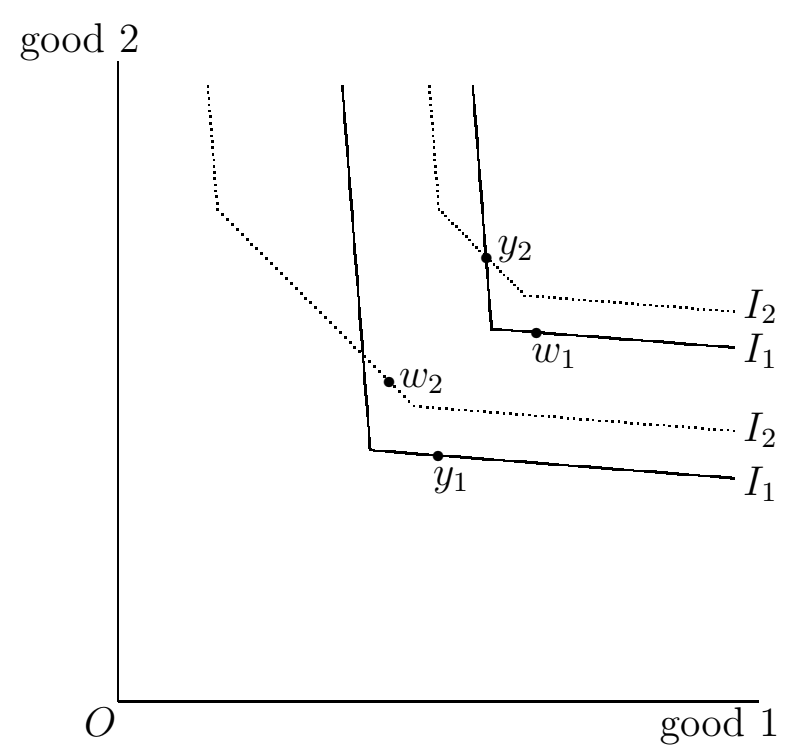

Figure 2: Allocations $y$ and $w$ are not egalitarian-equivalent.

have $z \succ y$. Similarly, by (2), $w \succ z$. Finally, from (4), (5) and (8) together, it must be true that $x \succ w$. Thus, the relation $\succ$ has a cycle.

Notice that in the above proof, we have considered two egalitarianequivalent allocations such that their reference bundles cannot be on one monotone path from the origin in the consumption space. In fact, this has caused the cycle in the social preference relation. If we restrict the range of egalitarian-reference bundles on a fixed monotone path (or for simplicity, some fixed ray) from the origin, then we have a positive result: there exists a social preference function satisfying Weak Pareto, P-Conditional $\bar{r}$-Egalitarian-Equivalence, and Transitivity. A good example is the social preference function due to Pazner and Schmeidler (1978).

The Pazner-Schmeidler function, $\boldsymbol{f}_{P S}$ : Fix a vector $\bar{r} \in \mathbb{R}_{++}^{m}$. For each $R_{N} \in \mathcal{R}, \succeq_{P S}=f_{P S}\left(R_{N}\right)$ is defined as follows: For each $i \in N$ and each $x_{i} \in \mathbb{R}_{+}^{m}$, let $\lambda_{i}\left(x_{i}\right) \in \mathbb{R}_{+}$be such that $x_{i} I_{i} \lambda_{i}\left(x_{i}\right) \bar{r}$. Then, for all $x, y \in X$, $x \succeq_{P S} y \Leftrightarrow \min _{i \in N} \lambda_{i}\left(x_{i}\right) \geq \min _{i \in N} \lambda_{i}\left(y_{i}\right)$. 
A refinement of the Pazner-Schmeidler function, which also satisfies the Pareto principle, can be obtained by lexicographic comparison of the vectors $\left(\lambda_{i}\left(x_{i}\right)\right)_{i \in N}$.

Recently, Fleurbaey (2001) has characterized the Pazner-Schmeidler function by the axioms, Transitivity, Weak Pareto, and "Minimal Equity for Proportional Allocations," ${ }^{5}$ which is a weakening of the equity concept due to Hammond (1976). Next, we present another simple characterization of the Pazner-Schmeidler function by the axioms, Quasi-Transitivity, Weak Pareto, and P-Conditional $\bar{r}$-Egalitarian-Equivalence. Note that there is no logical relation between P-Conditional $\bar{r}$-Egalitarian-Equivalence and Mimimal Equity for Proportional Allocations.

Proposition 3 The Pazner-Schmeidler function satisfies Weak Pareto, P-Conditional $\bar{r}$-Egalitarian-Equivalence, Transitivity, Completeness, and Continuity. If a social preference function satisfies Weak Pareto, PConditional $\bar{r}$-Egalitarian-Equivalence, and Quasi-Transitivity, then it is the Pazner-Schmeidler function or a refinement.

Proof. It is clear that the Pazner-Schmeidler function $f_{P S}$ satisfies Transitivity, Completeness, Continuity and Weak Pareto. We show that it satisfies P-Conditional $\bar{r}$-Egalitarian-Equivalence. Let $R_{N} \in \mathcal{R}^{n}$ and $\succeq_{P S}=f_{P S}\left(R_{N}\right)$. Let $x, y \in X$ be such that $x \nsucc_{P} y, y \nsucc_{P} x$, and $x \in E_{\bar{r}}$ but $y \notin E_{\bar{r}}$. We need to show that $x \succ_{P S} y$. If $x_{i} I_{i} y_{i}$ for all $i \in N$, then either $x, y \in E_{\bar{r}}$ or $x, y \notin E_{\bar{r}}$, which is a contradiction. Hence, there exists $h \in N$ with $x_{h} P_{h} y_{h}$. Then, $\lambda_{h}\left(x_{h}\right) \bar{r} I_{h} x_{h} P_{h} y_{h} I_{h} \lambda_{h}\left(y_{h}\right) \bar{r}$. By monotonicity of $R_{h}$, we have $\lambda_{h}\left(x_{h}\right)>\lambda_{h}\left(y_{h}\right)$. Since $x \in E_{\bar{r}}$, it follows that for all $i \in N, \lambda_{i}\left(x_{i}\right)=\lambda_{h}\left(x_{h}\right)$. Thus, $\min _{i \in N} \lambda_{i}\left(x_{i}\right)=\lambda_{h}\left(x_{h}\right)>\lambda_{h}\left(y_{h}\right) \geq \min _{i \in N} \lambda_{i}\left(y_{i}\right)$. Therefore, $x \succ_{P S} y$.

Next we prove the second part. Let $f$ be a social preference function satisfying Weak Pareto, P-Conditional $\bar{r}$-Egalitarian-Equivalence, and QuasiTransitivity. Let $R_{N} \in \mathcal{R}^{n}$ be given. We need to show that $\succ_{P S} \subset \succ$, that is, for all $x, y \in X$, if $x \succ_{P S} y$, then $x \succ y$. Suppose that $x \succ_{P S} y$. Then, $\min _{j \in N} \lambda_{j}\left(x_{j}\right)>\min _{j \in N} \lambda_{j}\left(y_{j}\right)$. We distinguish two cases.

\footnotetext{
${ }^{5}$ Minimal Equity for Proportional Allocations I: Fix $\Omega \in \mathbb{R}_{++}^{m}$. For all $x, y \in X$, if (i) for all $i \in N, x_{i}=\alpha_{i} \Omega$ and $y_{i}=\beta_{i} \Omega$ for some $\alpha_{i}, \beta_{i} \in \mathbb{R}_{+}$,

(ii) $W(x) \equiv\left\{i \in N \mid x_{i}=\min _{j \in N} x_{j}\right\}=\left\{i \in N \mid y_{i}=\min _{j \in N} y_{j}\right\}$ and $B(x) \equiv\{i \in N \mid$ $\left.x_{i}=\max _{j \in N} x_{j}\right\}=\left\{i \in N \mid y_{i}=\max _{j \in N} y_{j}\right\}$,

(iii) for all $i \in W(x), y_{i}<x_{i}$, and for all $i \in B(x), y_{i}>x_{i}$,

(iv) for all $k \in N \backslash[W(x) \cup B(x)], x_{k}=y_{k}$,

then $x \succeq y$.
} 
Case 1: $\max _{j \in N} \lambda_{j}\left(y_{j}\right)<\min _{j \in N} \lambda_{j}\left(x_{j}\right)$.

For all $i \in N, x_{i} I_{i} \lambda_{i}\left(x_{i}\right) \bar{r} \quad R_{i}\left[\min _{j \in N} \lambda_{j}\left(x_{j}\right)\right] \bar{r} P_{i}\left[\max _{j \in N} \lambda_{j}\left(y_{j}\right)\right] \bar{r} R_{i}$ $\lambda_{i}\left(y_{i}\right) \bar{r} I_{i} y_{i}$. By Weak Pareto, $x \succ y$.

Case $2: \max _{j \in N} \lambda_{j}\left(y_{j}\right) \geq \min _{j \in N} \lambda_{j}\left(x_{j}\right)$.

Let $\lambda^{*}=\frac{1}{2}\left[\min _{j \in N} \lambda_{j}\left(x_{j}\right)+\min _{j \in N} \lambda_{j}\left(y_{j}\right)\right]$. Note that $\min _{j \in N} \lambda_{j}\left(y_{j}\right)<\lambda^{*}<$ $\min _{j \in N} \lambda_{j}\left(x_{j}\right) \leq \max _{j \in N} \lambda_{j}\left(y_{j}\right)$. Define $z \in X$ by $z_{i}=\lambda^{*} \bar{r}$ for all $i \in N$. Then, for all $i \in N, x_{i} I_{i} \lambda_{i}\left(x_{i}\right) \bar{r} R_{i}\left[\min _{j \in N} \lambda_{j}\left(x_{j}\right)\right] \bar{r} P_{i} \lambda^{*} \bar{r}=z_{i}$. By Weak Pareto, $x \succ z$. On the other hand, let $h, k \in N$ be such that $\lambda_{h}\left(y_{h}\right)=$ $\max _{j \in N} \lambda_{j}\left(y_{j}\right)$ and $\lambda_{k}\left(y_{k}\right)=\min _{j \in N} \lambda_{j}\left(y_{j}\right)$. Then, $y_{h} I_{h} \lambda_{h}\left(y_{h}\right) \bar{r} P_{h} \lambda^{*} \bar{r}=z_{h}$ whereas $z_{k}=\lambda^{*} \bar{r} P_{k} \lambda_{k}\left(y_{k}\right) \bar{r} I_{k} y_{k}$. Thus, there is no Pareto domination relation between $y$ and $z$. Notice that $z$ is an $\bar{r}$-egalitarian-equivalent allocation, while $y$ is not because $\lambda_{h}\left(y_{h}\right)>\lambda_{k}\left(y_{k}\right)$. By P-Conditional $\bar{r}$-EgalitarianEquivalence, we have $z \succ y$. From Quasi-Transitivity, $x \succ y$.

Corollary 1 The Pazner-Schmeidler function is the unique social preference function that satisfies Weak Pareto, P-Conditional $\bar{r}$-EgalitarianEquivalence, Quasi-Transitivity, and Continuity.

Proof. The statement follows from the above proposition and Lemma 2 in Fleurbaey (2001).

If we adopt egalitarian-equivalence as the first criterion, and impose the Pareto principle only as the second criterion, then we can always construct transitive social preference relations. This holds true whether we restrict the range of egalitarian-reference bundles or not. However, there is also a serious drawback. With the equity-first principle, we cannot have continuous social preference relations.

Proposition 4 There exists a social preference function satisfying Egalitarian-Equivalence, E-Conditional Pareto, Completeness, and Transitivity. However, there does not exist a social preference function satisfying Egalitarian-Equivalence, E-Conditional Pareto, and Continuity together.

Proof. Let $f_{P S_{L}}$ be the lexicographic refinement of the Pazner-Schmeidler function. For each $R_{N} \in \mathcal{R}^{n}$, let $\succeq_{P S_{L}}=f_{P S_{L}}\left(R_{N}\right)$. Define a social preference function $f$ as follows: For each $R_{N} \in \mathcal{R}^{n}, \succeq=f\left(R_{N}\right)$ is defined by:

$\forall x, y \in X$,

(i) if $x \succ_{E} y$, then $x \succ y$; and

(ii) if $x \nsucc_{E} y$ and $y \nsucc_{E} x$, then $x \succeq y \Leftrightarrow x \succeq_{P S_{L}} y$. 
By (i), $f$ sataisfies Egalitarian-Equivalence. Since $f_{P S_{L}}$ satisfies Pareto, it follows from (ii) that $f$ satisfies E-Conditional Pareto. Because $f_{P S_{L}}$ satisfies Completeness, so does $f$. To check Transitivity of $f$, let $R_{N} \in \mathcal{R}^{n}$ be given, and let $x, y, z \in X$ be such that $x \succeq y$ and $y \succeq z$. We distinguish three cases. Case 1: $z \in E$.

From (i) and $y \succeq z$, we must have $y \in E$. By the same reasoning, $x \in E$. Hence, $x \succeq y$ and $y \succeq z$ hold only if $x \succeq_{P S_{L}} y$ and $y \succeq_{P S_{L}} z$. Because $\succeq_{P S_{L}}$ is transitive, $x \succeq_{P S_{L}} z$. By (ii), $x \succeq z$.

Case 2: $z \notin E$ and $y \in E$.

By the same argument as in Case $1, x \in E$. Then, from (i), we have $x \succ z$. Case 3: $z \notin E$ and $y \notin E$.

If $x \in E$, then by (i), $x \succ z$. If $x \notin E$, then $x \succeq y$ and $y \succeq z$ imply $x \succeq_{P S_{L}} y$ and $y \succeq_{P S_{L}} z$. Hence, $x \succeq_{P S_{L}} z$ and so $x \succeq z$.

Therefore, $f$ satisfies Transitivity.

Next, we prove the second part. Let $f$ be a social preference function satisfying Egalitarian-Equivalence and E-Conditional Pareto. We show that $f$ must violate Continuity. Let $R_{N} \in \mathcal{R}^{n}$ be such that $R_{i}=R_{j}$ for all $i, j \in N$. Let $\succeq=f\left(R_{N}\right)$. Define $x \in X$ by $x_{i}=(1, \ldots, 1) \in \mathbb{R}_{+}^{m}$ for all $i \in N$. Clearly, $x \in E$. Define a sequence of allocations $\left\{y^{t}\right\}$ by:

$\forall t=1,2, \ldots, \forall i \in N, y_{i}^{t}=\left(2+\frac{i}{t}, 2+\frac{i}{t}, \ldots, 2+\frac{i}{t}\right) \in \mathbb{R}_{+}^{m}$. Let $i, j \in N$ be such that $i>j$. For each $t$, because $y_{i}^{t} \gg y_{j}^{t}$ and $R_{i}=R_{j}$, there cannot exist $y_{0} \in \mathbb{R}_{+}^{m}$ such that $y_{i}^{t} I_{i} y_{0}$ and $y_{j}^{t} I_{j} y_{0}$. Thus, for all $t, y^{t} \notin E$. By Egalitarian-Equivalence, $x \succ y^{t}$ for all $t$. On the other hand, $\lim _{t \rightarrow \infty} y^{t}=z$ where $z=(2, \ldots, 2) \in \mathbb{R}_{+}^{m}$. It is clear that $z \in E$. Since $x, z \in E$ and $z \succ_{P} x$, by E-Conditional Pareto, we have $z \succ x$. This means that the set $\{w \in X \mid x \succeq w\}$ is not closed.

\section{Maximal Allocations in an Edgeworth Box}

In this section, we study the maximal elements in an Edgeworth box of social preference relations based on the efficiency-first principle or the equity-first principle with egalitarian-equivalence as the concept of equity. As Tadenuma (2002) showed, when no-envy is the concept of equity, the set of maximal elements of the coarsest efficiency-first relation is in general different from the set of maximal elements of the coarsest equity-first relation. More precisely, the former set is a proper subset of the latter set, and when individuals' preferences are not convex, the former set may be empty while the latter set is 
always non-empty. In contrast with these results, when we adopt egalitarianequivalence as the concept of equity, the set of maximal elements of the coarsest efficiency-first relation always coincides with that of the coarsest equity-first relation. Moreover, the set is non-empty even if individuals' preferences are not convex. The results hold true whether we restrict the range of reference bundles or not. (Hence, we only present the results for the case where no restriction is imposed on the range.) It should be noted that despite the fact that the efficiency-first relation may have a cycle when we impose no restriction on the range of reference bundles, and despite the fact that the equity-first relation lacks continuity, there exist maximal elements of these relations in the Edgeworth box.

The basic facts underlying these contrasting results are the following. Consider two selection procedures from an Edgeworth box. In the first procedure, we first choose the set of Pareto efficient allocations in the whole Edgeworth box, and secondly select the set of equitable allocations in the Pareto set. In the second procedure, by contrast, we first choose the set of equitable allocations in the whole Edgeworth box, and secondly select the set of allocations that are not Pareto-dominated by any equitable allocation. When no-envy is the concept of equity, the two sets obtained through these two procedures are in general different. However, if we adopt egalitarianequivalence as the concept of equity, then the two sets always coincide as the next lemma shows.

Fix $\Omega \in \mathbb{R}_{++}^{m}$ and let $Z=\left\{x \in X \mid \sum_{i \in N} x_{i} \leq \Omega\right\}$.

Lemma 1 For all $R_{N} \in \mathcal{R}^{n}, P(E(Z))=P(Z) \cap E(Z)$.

Proof. Let $R_{N} \in \mathcal{R}^{n}$ be given. If $x \in P(Z) \cap E(Z)$, then $x \in E(Z)$, and for all $y \in Z$, and in particular, all $y \in E(Z), y \nsucc_{P} x$. Hence, $x \in P(E(Z))$. Thus, $P(Z) \cap E(Z) \subset P(E(Z))$.

To show that $P(E(Z)) \subset P(Z) \cap E(Z)$, let $x \in P(E(Z))$. Obviously, $x \in E(Z)$. Suppose that $x \notin P(Z)$. Then, there exists $y \in Z$ with $y \succ_{P} x$. Since $R_{i}$ is strictly monotonic for all $i \in N$, there exists $z \in Z$ such that $z_{i} P_{i} x_{i}$ for all $i \in N$. Because $x \in E(Z)$, there is $a_{0} \in Z$ such that $x_{i} I_{i} a_{0}$ for all $i \in N$. Hence, $z_{i} P_{i} a_{0}$ for all $i \in N$. By continuity of $R_{i}$, there exist $\alpha>1$ such that $z_{i} P_{i} \alpha a_{0}$ for all $i \in N$. For each $i \in N$, there exists $\beta_{i}<1$ with $\beta_{i} z_{i} I_{i} \alpha a_{0}$. Define $\beta z \equiv\left(\beta_{1} z_{1}, \ldots, \beta_{n} z_{n}\right)$. Then, $\beta z$ is egalitarianequivalent with $\alpha a_{0}$ being the reference bundle. Since $z$ is feasible, so is $\beta z$. Thus, $\beta z \in E(Z)$. For all $i \in N, \beta_{i} z_{i} I_{i} \alpha a_{0} P_{i} a_{0} I_{i} x_{i}$. Hence, $\beta z \succ_{P} x$. This means that $x \notin P(E(Z))$, which is a contradiction. 
Proposition 5 If a social preference function $f$ satisfies

(i) Weak Pareto and P-Conditional Egalitarian-Equivalence, or

(ii) Egalitarian-Equivalence and E-Conditional Pareto,

then, for all $R_{N} \in \mathcal{R}^{n}, M(Z, \succeq) \subset P(Z) \cap E(Z)$.

Proof. Assume that a social preference function $f$ satisfies Weak Pareto and P-Conditional Egalitarian-Equivalence. Let $R_{N} \in \mathcal{R}^{n}$ be given. Let $x \in M(Z, \succeq)$. Suppose that $x \notin P(Z)$. Then there exists $y \in Z$ with $y \succ_{P} x$. Since $R_{i}$ is strictly monotonic for all $i \in N$, there exists $z \in Z$ such that $z_{i} P_{i} x_{i}$ for all $i \in N$. By Weak Pareto, $z \succ x$ and hence $x \notin M(Z, \succeq)$, which is a contradiction. Thus, $x \in P(Z)$. Suppose that $x \notin E(Z)$. From Pazner and Schmeidler (1978), there exists $y \in P(Z) \cap E(Z)$. Since both $x$ and $y$ are in $P(Z), x \nsucc_{P} y$ and $y \nsucc_{P} x$. Moreover, $y \succ_{E} x$. By P-Conditional Egalitarian-Equivalence, $y \succ x$. This contradicts $x \in M(Z, \succeq)$. Therefore, $x \in P(Z) \cap E(Z)$.

Next, assume that a social preference function $f$ satisfies EgalitarianEquivalence and E-Conditional Pareto. Let $R_{N} \in \mathcal{R}^{n}$ be given. Let $x \in$ $M(Z, \succeq)$. If $x \notin E(Z)$, then by Egalitarian-Equivalence, for any $y \in E(Z) \neq$ $\emptyset, y \succ x$, which contradicts $x \in M(Z, \succeq)$. Thus, $x \in E(Z)$. Suppose that $x \notin P(E(Z))$. Since $x \in E(Z)$, this holds true only if there exists $y \in E(Z)$ with $y \succ_{P} x$. Then, by E-Conditional Pareto, $y \succ x$, which is in contradiction with $x \in M(Z, \succeq)$. Thus, $x \in P(E(Z))$. By Lemma $1, x \in P(Z) \cap E(Z)$.

Let $R_{N} \in \mathcal{R}^{n}$ be given. Define the strict social preferece relation $\succ_{P E}$ as follows:

$\forall x, y \in X: x \succ_{P E} y \Leftrightarrow\left[x \succ_{P} y\right]$ or $\left[x \succ_{P} y, y \succ_{P} x\right.$ and $\left.x \succ_{E} y\right]$.

Then, define the social preference relation $\succeq_{P E}$ by

$\forall x, y \in X: x \succeq_{P E} y \Leftrightarrow y \nsucc_{P E} x$.

Define the social preference function $f_{P E}$ by $f_{P E}\left(R_{N}\right)=\succeq_{P E}$ for all $R_{N} \in$ $\mathcal{R}^{n}$. If a social preference function $f$ satisfies Weak Pareto and P-Conditional Egalitarian-Equivalence, then for all $x, y \in X, x \succ_{P E} y \Rightarrow x \succ y$. Hence, $f_{P E}$ is the coarsest social preference function that satisfies Weak Pareto and P-Conditional Egalitarian-Equivalence.

Similarly, given $R_{N} \in \mathcal{R}^{n}$, define $\succ_{E P}$ as follows:

$\forall x, y \in X: x \succ_{E P} y \Leftrightarrow\left[x \succ_{E} y\right]$ or $\left[x \succ_{E} y, y \succ_{E} x\right.$ and $\left.x \succ_{P} y\right]$.

Then, define $\succeq_{E P}$ by:

$\forall x, y \in X: x \succeq_{E P} y \Leftrightarrow y \nsucc_{E P} x$.

The social preference function $f_{E P}$ defined by $f_{E P}\left(R_{N}\right)=\succeq_{E P}$ is the 
coarsest social preference function satisfying Egalitarian-Equivalence and EConditional Pareto.

Proposition 6 For all $R_{N} \in \mathcal{R}^{n}, M\left(Z, \succeq_{P E}\right)=M\left(Z, \succeq_{E P}\right)=P(Z) \cap$ $E(Z)$.

Proof. Let $R_{N} \in \mathcal{R}^{n}$ be given. By Proposition 5, $M\left(Z, \succeq_{P E}\right) \subset P(Z) \cap$ $E(Z)$. To show that $P(Z) \cap E(Z) \subset M\left(Z, \succeq_{P E}\right)$, assume $x \in P(Z) \cap E(Z)$. Then, for all $y \in Z, y \nsucc_{P} x$, and since $x \in E(Z), y \nsucc_{E} x$. Hence, $y \nsucc_{P E} x$. Thus, $x \in M\left(Z, \succeq_{P E}\right)$. Therefore, $M\left(Z, \succeq_{P E}\right)=P(Z) \cap E(Z)$.

Next, we show that $P(E(Z)) \subset M\left(Z, \succeq_{E P}\right)$. Assume $x \in P(E(Z))$. Let

$y \in Z$. Since $x \in E(Z)$, we have $y \nsucc_{E} x$. If $x \nsucc_{E} y$, then $y$ is also in $E(Z)$, and because $x \in P(E(Z)), y \nsucc_{P} x$. Hence, $y \nsucc_{E P} x$. It follows that $x \in M\left(Z, \succeq_{E P}\right)$. Thus, $P(E(Z)) \subset M\left(Z, \succeq_{E P}\right)$.

By Proposition 5 and Lemma 1 , we have that $M\left(Z, \succeq_{E P}\right)=P(Z) \cap E(Z)$.

\section{Concluding Remarks}

In the literature of fair allocation theory, many authors have investigated the existence and properties of allocations that are egalitarian-equivalent and Pareto efficient in the whole feasible set. However, the concept of egalitarianequivalence is also useful to construct reasonable social preference relations over all allocations. As we have shown, the properties of social preference functions reflecting the idea of equity are much different depending on (i) to which criterion we give priority, Pareto or equity-as-egalitarian-equivalence, and (ii) how we restrict the range of egalitarian-reference consumption bundles if Pareto is given priority.

As for the maximal allocations, however, there arises no essential difference between the two principles. The set of maximal allocations of the coarsest efficiency-first social preferences and that of the coarsest equity-first social preferences coincide, and the set of maximal allocations of any social preferences based on either the efficiency-first principle or the equity-first principle is a subset of this set. This result is in marked contrast with the case where no-envy is adopted as the concept of equity.

In the problem of distributing fixed amounts of social endowments of commodities, Pazner and Schmeidler (1978), Fleurbaey and Maniquet (1996, 
2001) and Fleurbaey (2001) make ethical arguments to justify taking reference consumption bundles proportional to the vector of social endowments. Our results show that if we would like not only to identify the "optimal" allocations but also to construct acyclic social preference relations over all allocations, respecting the efficiency-first and equity-second principle, then it is logically inevitable to take one monotone path from the origin along which reference bundles are taken. Further investigation for ethical principles to justify a particular monotone path of reference bundles, which may depend on the features of each economic model, should be left for future researches.

\section{References}

Arrow, K. J. (1951), Social Choice and Individual Values, New York: Wiley. Second ed., 1963.

Feldman, A. M. and A. Kirman (1974), "Fairness and Envy," American Economic Review 64, 996-1005.

Fleurbaey, M. (2001), "The Pazner-Schmeidler Social Ordering: A Defense," mimeo., Université de Pau.

Fleurbaey, M. and F. Maniquet (1996), Utilitarianism versus Fairness in Welfare Economics, forthcoming in M. Salles and J. A. Weymark (eds.), Justice, Political Liberalism and Utilitarianism: Themes from Harsarnyi and Rawls, Cambridge: Cambridge University Press.

Fleurbaey, M. and F. Maniquet (2001), "Fair Social Orderings," mimeo., Université de Pau.

Foley, D. (1967), "Resource Allocation and the Public Sector," Yale Economic Essays 7, 45-98.

Gärdenfors, P. (1978), "Fairness without Interpersonal Comparisons," Theoria 44, 57-74.

Hammond, P. J. (1976), "Equity, Arrow's Conditions and Rawls' Difference Principle," Econometrica 44, 793-804.

Kolm, S.-C. (1971), Justice et Equité, CEPREMAP, Paris. 
Pazner, E. A. and D. Schmeidler (1978), "Egalitarian Equivalent Allocations: A New Concept of Economic Equity," Quarterly Journal of Economics 92, 671-687.

Suzumura, K. (1981a), "On Pareto-Efficiency and the No-Envy Concept of Equity," Journal of Economic Theory 25, 367-379.

Suzumura, K. (1981b), "On the Possibility of "Fair" Collective Choice Rule," International Economic Review 22, 351-364.

Suzumura, K. (1996), "Interpersonal Comparisons of the Extended Sympathy Type and the Possibility of Social Choice," in K. J. Arrow, A. Sen and K. Suzumura (eds.), Social Choice Re-examined, Volume 2, 202-229, IEA, London: Macmillan Press.

Tadenuma, K. (1998), "Efficiency-First or Equity-First? Two Principles and Rationality of Social Choice," Discussion Paper Series No. 1998-01, Hitotsubashi University (http://wakame.econ.hitu.ac.jp/ ^koho/4links/dp-abstract.htm\#9801).

Tadenuma, K. (2002), "Efficiency-First or Equity-First? Two Principles and Rationality of Social Choice," Journal of Economic Theory 104, $462-472$ 